\title{
alloSCT beim rezidivierten follikulären Lymphom: Chance mit Risiko
}

Im Rezidiv nach autologer Stammzelltransplantation (ASCT) gibt es für Patienten mit follikulärem Lymphom (FL) verschiedene Therapieoptionen, aber keinen Standard. Eine Option ist die allogene Stammzelltransplantation mit reduzierter Intensität (RICalloSCT).
Monaten. Die 5-Jahres-Rate für Rezidive oder Progress betrug $16 \%$. Rezidive traten meist im ersten Jahr nach RICalloSCT auf, in einem Fall aber auch erst nach 90 Monaten. Die progressionsfreie Überlebensrate nach 5 Jahren betrug $48 \%$ und die 5-Jahres-Gesamtüberlebensrate $51 \%$. Als Einflussfaktoren, die mit einem schlechteren Therapieergebnis assoziiert waren, wurden ein Alter über 45 Jahre und eine chemorefraktäre Erkrankung identifiziert. Einige Patienten erhielten nach Rezidiv eine DonorLymphozyten-Infusion. Auch hier konnte noch einmal teilweise eine anhaltende Remission erreicht werden.

Die kumulative Inzidenz einer akuten Graft-versus-Host-Erkrankung (GvHD) betrug an Tag 100 sowie an Tag 180 nach der Transplantation $45 \%$. Die Rate der chronischen GvHD lag nach 2 Jahren bei $51,2 \% .27$ Patienten erkrankten an sekundären Neoplasien, 6 verstarben daran.

Fazit: Die RICalloSCT kann eine wirksame Salvage-Therapie für Patienten mit FL sein, die nach mehreren Therapielinien und einer ASCT ein Rezidiv erleiden. In dieser Situation scheint immer noch eine Heilung möglich, allerdings mit erheblichem therapiebedingtem Mortalitätsund Malignomrisiko. Weniger toxische Regimes wären daher wünschenswert, und die Forscher verweisen in diesem Zusammenhang auf Studien zu neuen zielgerichteten Therapien. Friederike Klein

Robinson SP et al. Reduced intensity allogeneic stem cell transplantation for follicular lymphoma relapsing after an autologous transplant achieves durable long term disease control. An analysis from the Lymphoma Working Party Of the EBMT. Ann Oncol. 2016;27(6):1088-94.

\section{Indolentes Non-Hodgkin-Lymphom: Zwei Anti-CD20-Therapien hintereinander}

\author{
Kontrolliert eine Rituximab-basierte Therapie ein indolentes Non-Hodgkin- \\ Lymphom (iNHL) nicht ausreichend, bleiben nur wenige Optionen. Eine \\ Möglichkeit könnte der CD20-Antikörper Obinutuzumab darstellen, der auch \\ bei Rituximab-refraktären Lymphomen Wirksamkeit gezeigt hat.
}

\footnotetext{
$\mathrm{n}$ der Phase-III-Studie GADOLIN wurden Patienten mit Rituximab-refraktärem iNHL mit Obinutuzumab plus Bendamustin oder Bendamustin allein behandelt. Insgesamt 396 Patienten mit CD20-positivem iNHL erhielten randomisiert eine Induktionstherapie mit $6 \mathrm{Zy}$ klen (q28d) Obinutuzumab plus Bendamustin (Obinutuzumab: $1.000 \mathrm{mg}$ i.v. an den Tagen 1,8 und 15 von Zyklus 1 sowie an Tag 1 der Zyklen 2-6; Bendamustin: $90 \mathrm{mg} / \mathrm{m}^{2}$ i.v. an den Tagen 1 und 2 der Zyklen 1-6) oder Bendamustin allein (Bendamustin: $120 \mathrm{mg} / \mathrm{m}^{2}$ i.v. an den Tagen 1 und 2 der Zyklen 1-6). Zeigte sich kein Progress, erfolgte in der Kombinationsgruppe bis zu 2 Jahre lang eine Erhal-
}

tungstherapie mit Obinutuzumab (1.000 mg i.v. alle 2 Monate).

Nach einer geplanten Interimsanalyse wurde die Studie wegen der Überlegenheit des Kombinationsarms gestoppt. Nach einem medianen Follow-up von 21,9 Monaten in der Kombinations- und 20,3 Monaten in der Monotherapiegruppe war das progressionsfreie Überleben (PFS) in der Kombinationsgruppe noch nicht erreicht, während es im Bendamustin-Arm bei 14,9 Monaten lag (Hazard Ratio 0,$55 ; \mathrm{p}=0,0001$ ).

132 (68\%) der Patienten in der Kombinationsgruppe und $123(62 \%)$ in der Bendamustin-Gruppe entwickelten Nebenwirkungen vom Grad 3-5, am häufigsten
Neutropenien (33 vs. $26 \%$ ), Thrombozytopenien (11 vs. $16 \%$ ), Anämien (8 vs. $10 \%)$ und Infusionsreaktionen (11 vs. $6 \%$ ). Schwere unerwünschte Ereignisse traten bei $38 \%$ der Patienten in der Obinutuzumab/Bendamustin-Gruppe und bei $33 \%$ in der Bendamustin-Gruppe auf, solche mit Todesfolge bei jeweils $6 \%$. $25 \%$ der Todesfälle bei Kombinationsund $42 \%$ bei Monotherapie wurden als behandlungsassoziiert eingestuft.

Fazit: Die Kombination von Obinutuzumab/Bendamustin gefolgt von einer Obinutuzumab-Erhaltung zeigt bei Patienten mit gegen Rituximab refraktären iNHL bessere Wirksamkeit als Bendamustin alleine. Die Toxizität der Kombination scheint beherrschbar und kaum höher als unter Bendamustin-Monotherapie.

Friederike Klein

Sehn LH et al. Obinutuzumab plus bendamustine versus bendamustine monotherapy in patients with rituximab-refractory indolent non-Hodgkin lymphoma (GADOLIN): a randomised, controlled, open-label, multicentre, phase 3 trial. Lancet Oncol. 2016;17(8):1081-93. 